\title{
Evaluación de la Eficacia de un Programa Preventivo Para la Depresión en Adolescentes de Sexo Femenino
}

\author{
Evaluation of The Efficacy of a Prevention Program for Depression in Female \\ Adolescents
}

\author{
Félix Cova \\ Universidad de Concepción, Chile \\ Paulina Rincón \\ Universidad de Concepción, Chile \\ $\&$ \\ Roberto Melipillán \\ Universidad del Desarrollo, Chile
}

(Rec: 29 de Octubre de 2009 / Acep: 24 de Agosto de 2011)

\begin{abstract}
Resumen
Se evalúa el efecto de un programa preventivo de depresión aplicado a adolescentes de sexo femenino que cursaban primero medio. Este programa fue implementado en dos modalidades: 1) Indicada, aplicado a participantes con sintomatología depresiva subumbral (grupo experimental $\mathrm{n}=101$; grupo control $\mathrm{n}=108$ ), $\mathrm{y}$ 2) Universal, aplicado a cursos completos sólo de niñas (grupo experimental=119 participantes; grupo control $\mathrm{n}=118$ ). El programa tuvo 11 sesiones, de 1,5 hora de duración, con 15 a 23 participantes por grupo. Se evaluó el efecto del programa en depresión, en ansiedad, conductas de autodaño y conductas externalizadas. El programa no mostró efectos en ninguna de sus dos modalidades de implementación. La modalidad indicada fue más satisfactoria para las participantes. Se comentan las limitaciones del estudio y sus proyecciones para el desarrollo de programas preventivos.
\end{abstract}

Palabras Clave: Depresión, prevención indicada, prevención universal, adolescentes.

\begin{abstract}
This study assesses the impact of a preventive program of depression in female adolescents from first grade of secondary school. The program was implemented in two ways: 1) Indicated: Applied to patients with subthreshold depressive symptoms (experimental group $\mathrm{N}=101$; control group $\mathrm{N}=108$ ) 2) Universal applied to a class comprised only by girls (experimental group $=119$; control group $\mathrm{N}=118$ ). The program had 11 sessions, that lasted 1,5 hours each, with 15 to 23 participants per group. The impact of the program was measured in terms of anxiety, self-damage behavior, and externalized behavior. The program did not show effects in any of the two modalities. The Indicated modality obtained more satisfaction in the group of participants. The limitations of the study and implications for preventive programs in mental health are discussed. Key words: Depression, indicated prevention, universal prevention, adolescent.
\end{abstract}

\footnotetext{
Este artículo fue realizado en el marco del proyecto FONDECYT INICIACIÓN Nº11060059.

Correspondencia: Félix Cova Solar, Facultad de Ciencias Sociales, Departamento de Psicología, Universidad de Concepción, Barrio Universitario s/n, Concepción, Chile. E-mail: fecova@ udec.cl
} 


\section{Introducción}

Desde la perspectiva de la salud mental pública uno de los desafíos más relevantes es enfrentar el tema de la depresión, en particular, desde una perspectiva preventiva (Muñoz, Cuijpers, Smit, Barrera \& Leykin, 2010). Con este propósito, se han desarrollado y evaluado, en especial en países de alto desarrollo económico, diversos programas que han mostrado resultados de interés (Calear \& Christensen, 2010; Merry, Mc Dowell, Hetrick, Bir \& Muller, 2003).

Buena parte de la investigación en este ámbito se ha desarrollado en niños, niñas y adolescentes tempranos intentando evitar el acentuado incremento de la depresión que se observa a partir de la adolescencia. Es importante destacar que aunque este incremento se observa en ambos géneros, es mucho más acentuado en las adolescentes (Cova, 2005; Zahn-Waxler, Crik, Shirtcliff \& Woods, 2006, citados en Cichetti \& Cohen, 2006).

Los programas preventivos que se han desarrollado en su mayoría tienen su fundamento en las teorías cognitivoconductuales de la depresión (Cova, Aburto, Sepúlveda \& Silva, 2006). El tratamiento cognitivo-conductual de la depresión supone que la persona desarrolle ciertas habilidades y formas de comportamiento que reducirían la experiencia depresiva. En la medida que se ha demostrado la eficacia de esta forma de terapia, se ha supuesto que estas habilidades y comportamientos podrían ser enseñados antes de que la persona se deprima, lo que en principio evitaría -o haría menos probable- el desarrollo de un episodio depresivo. Con esta base clínica, los programas se han centrado en actuar sobre las variables de riesgo y protección de tipo individual, fundamentalmente a través de talleres de entrenamiento destinados a reestructurar cogniciones negativas, enseñar un mejor manejo de la ansiedad y desarrollar habilidades efectivas de afrontamiento y resolución de problemas.

La evaluación de la eficacia de los programas implementados no muestra resultados claros. Los programas focalizados en población de riesgo (prevención selectiva) y/o con sintomatología subumbral (prevención indicada) muestran una tendencia a presentar efectos preventivos más nítidos que los programas universales (Calear \& Christensen, 2010; Collins \& Dozois, 2008; Horowitz \& Garber, 2006; Merry et al., 2003). Un factor que dificulta una valoración apropiada de la eficacia de los programas es que pocos estudios tienen un seguimiento suficiente como para observar la perdurabilidad de los efectos, si los hay, o posibles efectos latentes o tardíos (Sawyer et al., 2010).

En nuestro país existen pocos estudios de la eficacia o efectividad de intervenciones preventivas en este ámbito. La presente investigación evalúa el efecto de un programa preventivo en adolescentes de sexo femenino aplicado cuando éstas cursaban primero medio.

\section{Método}

\section{Participantes}

Las participantes fueron alumnas que cursaban primero medio en nueve liceos municipales o particulares subvencionados de la Provincia de Concepción. El programa fue dirigido sólo a niñas. Existieron dos condiciones diferentes para la selección de la muestra y la implementación del programa.

1. Modalidad indicada: En siete liceos se implementó esta modalidad. Para ello se realizó un tamizaje con el Inventario de Depresión de Beck, Segunda Versión (BDI-II) (Beck, Steer \& Brown, 1996). Las niñas que obtuvieron un puntaje igual o superior a 7 puntos fueron asignadas al azar al grupo experimental $(\mathrm{N}=101)$ o al grupo control $(\mathrm{N}=108)$.

2. Modalidad universal: Esta modalidad se implementó en dos liceos de niñas. Todas las niñas de los cursos seleccionados (un curso en un liceo, dos cursos en el otro) recibieron el programa. En total, 119 niñas corresponden al grupo experimental de esta modalidad. La misma cantidad de cursos por liceo fueron seleccionadas al azar como grupos controles. De esta forma el grupo control quedó conformado por 118 participantes.

Todas las participantes expresaron su consentimiento informado para participar de la investigación. Los padres también fueron informados y se les solicitó su consentimiento. Hubo solo un rechazo. Las adolescentes que cumplían con los criterios de presencia de trastorno depresivo en el último mes (22 de 319; aprox. 7\% del total) fueron incluidas en las intervenciones pero no fueron consideradas en los análisis y no están consideradas en el número de participantes descrito. Quienes cumplieron estos criterios fueron contactadas en forma confidencial y se les indicó la posible presencia de un problema que podría requerir atención profesional, ofreciéndoseles apoyo para obtener ésta.

\section{Programa Preventivo:}

Se usó como referente para el diseño de la intervención el Adolescent Coping with Stress Course (Clark \& Lewinsohn, 1995), el APEX Program (Gillham et al., 1992), y el Resourceful Adolescent Program (Shochet, Dadds, Holland, Whitefield, Harnett \& Osgarby, 2001). La versión definitiva del programa que se aplicó a las participantes fue de 11 sesiones de 1 hora y 30 minutos de duración cada una. En el caso de un liceo, éste debió ser adaptado realizándose un mayor número de sesiones más cortas. El programa se diseñó de modo que las participantes comprendieran la relación entre emociones, cogniciones y conductas, y fortalecieran una serie de habilidades: afrontamiento de problemas, comunicación, manejo de pensamientos y emociones negativas. Se generaron espacios para analizar 
y poner en práctica estas habilidades en relación a problemáticas habituales de la adolescencia, en particular en las relaciones con la pareja y amigos. La implementación de las actividades fue lograda, de acuerdo al registro de los propios facilitadores, en un promedio de $85 \%$.

\section{Instrumentos}

La evaluación de la sintomatología depresiva fue realizada a través del Inventario de Depresión de Beck-Segunda Versión (Beck et al., 1996; Melipillán, Cova, Rincón \& Valdivia, 2008). En la presente investigación obtuvo un valor alfa de 0,91 .

La identificación inicial de presencia de trastorno depresivo se realizó a través de la aplicación de la entrevista estructurada DISC-IV (Bravo et al., 2001).

Se evaluó también sintomatología ansiosa a través de Inventario de Ansiedad de Beck (BAI) (Beck \& Steer, 1993; Sanz \& Navarro, 2003) y se empleó un cuestionario ad hoc para evaluar conductas autolesivas y externalizantes.

Para evaluar estilo inferencial negativo se empleó el Adolescent Cognitive Style Questionaire, ACSQ (versión abreviada del instrumento) (Hankin \& Abramson, 2002). El instrumento mide las tres dimensiones del estilo inferencial negativo: atribución de causalidad (estabilidad y globalidad), inferencias negativas sobre las consecuencias e implicancias negativas hacia sí mismo. El alfa de Cronbach observado en esta investigación fue de 0,85.

Para evaluar estilo de afrontamiento rumiativo se utilizó la adaptación al español de la versión abreviada de la Ruminative Response Scale RRS (Cova, Rincón \& Melipillán, 2007), que permite evaluar dos componentes diferentes de la rumiación: reflexión y rumiación negativa. El alfa de Cronbach observado fue de 0,73 y 0,78 , respectivamente.

Autoestima fue evaluada con una versión resumida de la escala de Rosenberg (1965). El alfa de Cronbach observado en la presente investigación es de 0,89 para la escala total.

La satisfacción de las participantes con el programa de intervención fue evaluada con un cuestionario con formato de respuesta tipo Likert construido ad hoc. De igual forma fue evaluada la percepción de las usuarias respecto de si la participación en el programa había tenido algún efecto negativo para ellas.

\section{Procedimiento}

La intervención fue realizada por un equipo de licenciados en Psicología adecuadamente capacitados. Se desarrolló en un espacio físico proporcionado por los propios liceos. Aun cuando el compromiso inicial fue que se realizaría en horario de clases ello no fue factible en la mayoría de los Liceos, teniendo las niñas que retirarse algo más tarde de su jornada habitual o asistir en jornada alterna. En el caso de la aplicación del programa a cursos completos en la modalidad universal, se dividió al curso en dos grupos en espacios separados. Cada grupo, tanto de la modalidad universal como indicada, fue conducido por dos facilitadores. El número mínimo de integrantes originales de los grupos fluctuó entre 15 y 23.

Se realizó evaluación pre-test y evaluación a los siete meses de finalizado el programa.

\section{Análisis de datos}

Para la evaluación del efecto de la implementación del programa, se realizó un análisis de varianza controlando por el puntaje inicial de depresión en el grupo experimental y grupo control. Este análisis se realizó para cada modalidad de implementación del programa y se repitió con cada una de las demás variables consideradas, controlando por el puntaje inicial de cada variable.

\section{Resultados}

Línea base: la comparación del grupo experimental y control en las variables iniciales en ambas modalidades sólo mostró una diferencia significativa. Ésta se observó en la modalidad indicada, donde el grupo control mostró un promedio de conducta de autodaño superior (M GC indica$\mathrm{do}=0,85 ; \mathrm{M} \mathrm{GE}$ indicado $=0,41 ; \mathrm{t}=2,65 \mathrm{gl}=180 \mathrm{p}<0,01)$. No se observaron diferencias en las variables iniciales entre las participantes que no pudieron ser evaluadas nuevamente y aquellas que sí lo fueron.

La media de asistencia fue de 8,86 sesiones a la modalidad universal. Un 76,5\% de las participantes asistió a 8 sesiones o más. Sólo 4 participantes asistieron a menos de 6 sesiones $(3,4 \%)$. En la modalidad indicada la media de asistencia fue de 6 sesiones. Un $43 \%$ asistió a menos de 6 sesiones (un 8,9\% de las participantes no asistió a ninguna sesión). Dada la mayor inasistencia en estos grupos, el tamaño de ellos fue menor que en la modalidad universal. Estas diferencias son estadísticamente significativas ( $\mathrm{t}=6,94$ gl $149,57 \mathrm{p}<0,000$ ).

En la Tabla 1 se presentan los resultados de la evaluación de la participación en el programa de las niñas de ambas modalidades. Para facilitar la interpretación además del promedio de puntajes en relación a cada afirmación (rango $0-5$, donde 5 es el máximo desacuerdo), se presentan los porcentajes de acuerdo o desacuerdo dicotomizados.

La Tabla 2 presenta el puntaje pre y post test de las variables evaluadas en ambas modalidades en el grupo experimental y control. El valor F corresponde al análisis de covarianza realizado. La medición pre-test fue ingresada como covariable. Se presenta el valor original de las variables, aunque para los análisis éstas fueron normalizadas. 
Tabla 1: Evaluación del programa recibido por las participantes

\begin{tabular}{|c|c|c|c|c|c|c|c|c|c|c|}
\hline & \multicolumn{4}{|c|}{ Prevención indicada $\mathrm{N}=81$} & \multicolumn{4}{|c|}{ Prevención universal N=116 } & & \\
\hline & $\begin{array}{c}\text { De acuerdo } \\
\text { o Muy de } \\
\text { acuerdo }\end{array}$ & $\begin{array}{c}\text { Desacuerdo } \\
\text { o Muy en } \\
\text { desacuerdo }\end{array}$ & $X$ & DS & $\begin{array}{l}\text { De acuerdo } \\
\text { o Muy de } \\
\text { acuerdo }\end{array}$ & $\begin{array}{c}\text { Desacuerdo } \\
\text { o Muy en } \\
\text { desacuerdo }\end{array}$ & $X$ & DS & gl & $\mathrm{t}$ \\
\hline Le gustó participar & $71,6 \%$ & $8,7 \%$ & 4,09 & 1,06 & $64,1 \%$ & $16,6 \%$ & 3,77 & 1,27 & 203 & $1,94 * * *$ \\
\hline Le fue útil & $79,0 \%$ & $13,5 \%$ & 4,13 & 1,17 & $55,4 \%$ & $25,4 \%$ & 3,46 & 1,40 & 203 & $3,71 *$ \\
\hline Fue entretenido & $86,6 \%$ & $7,4 \%$ & 4,36 & 0,77 & $67,5 \%$ & $15,3 \%$ & 3,84 & 1,20 & 203 & $3,41 * * *$ \\
\hline Volvería a participar & $80,3 \%$ & $8,6 \%$ & 4,23 & 1,08 & $55,8 \%$ & $23,4 \%$ & 3,54 & 1,42 & 203 & $3,95 * * *$ \\
\hline Recibió burlas & $23,4 \%$ & 66,7 & 2,20 & 1,49 & $6,7 \%$ & $72,0 \%$ & 1,61 & 1,07 & 203 & $3,20 * *$ \\
\hline Problemas por participar & $7,4 \%$ & $79,0 \%$ & 1,68 & 1,07 & $5,9 \%$ & $63,0 \%$ & 1,57 & 1,00 & 203 & 0,74 \\
\hline
\end{tabular}

${ }^{a}$ Los porcentajes no suman $100 \%$ porque no están incluidas las respuestas de las participantes que respondieron "ni de acuerdo ni en desacuerdo".

*: $p<0,05 ;{ }^{* * *}: p<0,001$

Tabla 2: Valores pre y postest en la modalidad indicada y universal

\begin{tabular}{lcccccc}
\hline & \multicolumn{2}{c}{ Modalidad Indicada } & F & \multicolumn{2}{c}{ Modalidad Universal } & F \\
& Experim. & Control & & Experim. & Control & \\
\hline N pretest & 101 & 108 & & 119 & 118 & \\
N post-test & 82 & 96 & & 92 & 84 & \\
Depresión pre-test & 17,85 & 16,80 & & 15,51 & 13,80 & \\
Depresión post-test & 14,89 & 13,38 & 0,06 & 11,64 & 11,63 & 1,58 \\
Ansiedad pre-test & 14,44 & 13,14 & & 10,28 & 12,71 & \\
Ansiedad post-test & 16,32 & 14,47 & 0,14 & 9,49 & 11,41 & 0,04 \\
Estilo inferencial pre-test & 2,38 & 2,32 & & 2,08 & 1,98 & \\
Estilo inferencial post-test & 2,18 & 2,16 & 0,10 & 1,81 & 1,85 & 3,44 \\
Autodaño pre-test & 0,86 & 0,42 & & 0,47 & 0,75 & \\
Autodaño post-test & 0,79 & 0,24 & $4,19 *$ & 0,63 & 0,93 & 2,03 \\
Exter. pre-test & 5,20 & 4,79 & & 5,94 & 5,55 & \\
Exter. post-test & 5,29 & 4,60 & 1,94 & 5,19 & 4,53 & 1,94 \\
Reflexión pretest & 8,79 & 8,53 & & 8,36 & 8,23 & \\
Reflexión postest & 8,39 & 7,67 & 0,53 & 8,46 & 6,40 & $11,99 * * *$ \\
Rumiación negativa pre-test & 11,08 & 10,45 & & 9,74 & 9,72 & \\
Rumiación negativa post-test & 9,57 & 9,17 & 0,02 & 8,03 & 7,19 & 1,86 \\
Autoestima global pretest & 20,74 & 20,74 & & 21,53 & 21,59 & \\
Autoestima global post-test & 21,98 & 21,65 & 0,58 & 22,72 & 22,74 & 0,02 \\
Autoestima corporal pretest & 8,33 & 8,09 & & 8,55 & 8,76 & \\
Autoestima corporal postest & 8,47 & 8,47 & 0,08 & 8,96 & 9,40 & 0,70 \\
\hline
\end{tabular}

*: $p<0,05 ;{ }^{* * *}: p<0,001$

\section{Discusión}

El programa preventivo implementado para esta investigación consideró los elementos conceptuales y prácticos que están en la base de los principales programas en este ámbito que se han desarrollado en el mundo que, en su mayoría, emplean de modo anticipatorio, las estrategias y técnicas desarrolladas desde los enfoques cognitivo-conductuales para el tratamiento de la depresión (Collins \& Dozois, 2006).

La implementación de estos programas ha mostrado efectos positivos en algunos estudios, sin que, sin embargo, los resultados sean suficientemente concluyentes (Garber, 2008; Gladstone, Beardslee \& O'Connor, 2011; Shochet et al., 2001). De allí el interés de evaluar el efecto de un 
programa de esta naturaleza en adolescentes chilenas, más aún teniendo presente que las investigaciones realizadas hasta ahora han sido realizadas fundamentalmente en países muy distintos al nuestro.

Otro aspecto en que a la fecha no hay resultados claros es respecto de si la prevención universal o selectiva debiera ser la estrategia privilegiada en el caso de problemas como la depresión -aunque se ha observado una tendencia de los programas selectivos indicados a ser más efectivos- (Calear \& Christensen, 2010; Collins \& Dozois, 2008; Merry et al., 2003; Horowitz \& Garber, 2006). El presente estudio se planteó comparar ambas modalidades aunque sólo en población adolescente de sexo femenino. El desarrollo de estrategias preventivas focalizadas en población femenina ha sido destacada por diversos autores como una necesidad relevante (Le, Muñoz, Ippen \& Stoddard, 2003). Existen asimismo indicios de que programas que trabajan con grupos exclusivamente de mujeres podrían ser más útiles y satisfactorios para las participantes que los programas mixtos (Chaplin et al., 2006).

Los resultados obtenidos en la presente investigación acentúan las dudas existentes respecto de que las estrategias preventivas habituales que se están empleando en el campo de la prevención de la depresión en adolescentes sean las más pertinentes y adecuadas. El programa implementado no mostró efectos en ninguna de las modalidades. Estos efectos fueron analizados a sólo 7 meses de haber finalizado la implementación del programa, por lo que no puede atribuirse la falta de éstos a un excesivo tiempo transcurrido desde la finalización de la intervención.

La única variable donde se observaron diferencias entre el grupo experimental y control que indica un posible efecto positivo de la participación en el programa, fue en relación al componente de reflexión de la rumiación, referida al análisis de las causas de un problema vivido (observadas sólo en el grupo experimental indicado). Este eventual efecto podría estar relacionado con el énfasis del programa en el desarrollo de estrategias activas y positivas de solución de problemas de parte de las participantes como opuesto a estrategias rumiativas. Sin embargo, habría sido más esperable un efecto respecto de la rumiación negativa, que es más propiamente una estrategia pasiva de pensamiento repetitivo acerca del malestar que se experimenta, que respecto de la reflexión (Treynor, Gonzalez \& Nolen-Hoeksema, 2003).

Se observaron diferencias también, pero en un sentido negativo, entre el grupo experimental y control en la modalidad indicada respecto a las conductas de autodaño. Pese a la asignación al azar al grupo indicado y al grupo control, en este aspecto se observaron diferencias previas al inicio del programa entre ambos grupos. La existencia de diferencias en esta variable finalizado el programa, controladas las diferencias preexistentes, sugieren un posible efecto iatrogénico de la participación en éste, aunque el eventual efecto observado fue marginal (eta $\left.{ }^{2}: 0,02\right)$.
Si bien las investigaciones hasta ahora no han mostrado efectos iatrogénicos de la participación en programas de este tipo, es algo que requiere atención, particularmente en programas selectivos. Reunir participantes identificados por su condición de riesgo de depresión podría potenciar determinados aspectos negativos entre ellos. Sin embargo, dado que éste es un resultado aislado y no convergente con la investigación existente hasta ahora, es necesario obtener más evidencias antes de poder extraer alguna conclusión.

El presente estudio evaluó también la satisfacción de las participantes con el programa y la percepción de algún efecto de estigma o consecuencia negativa percibida por las participantes por participar en él. Al respecto, se observó mayor satisfacción en las participantes de la modalidad indicada. Es probable que ello tenga fundamentalmente relación con que en esa modalidad las adolescentes debían salir de su sala de clases para asistir al programa o incluso asistir en un horario especial, por lo que su participación era voluntaria; en cambio, en la modalidad universal, el programa se implementaba con el curso completo en un horario fijado por el establecimiento educacional para ello, lo que no permitía elegir participar o no en las sesiones. De hecho, 9 participantes que eran parte del grupo experimental indicado nunca asistieron. Otro factor que puede haber influido en la mayor satisfacción en la modalidad indicada es que la menor asistencia hizo que los grupos de intervención fueran más pequeños. Rappee et al. (2006) también observaron mayor satisfacción en adolescentes que recibieron un programa indicado respecto a uno universal. La contrapartida de esta mayor satisfacción es que casi un cuarto de las adolescentes de la modalidad indicada señala haber sido molestada por su participación en la programa, lo que en la modalidad universal sólo fue señalado por un $6 \%$ de ellas. Los riesgos de estigmatización de las modalidades indicadas de prevención si bien han sido destacados por diversos autores, no han sido muy estudiados. El estudio de Rapee et al. (2006) observó mayor estigmatización en un grupo indicado de prevención de la depresión en adolescentes respecto al universal, pero poco acentuados. En síntesis, los resultados obtenidos señalan que la modalidad indicada es más satisfactoria para las participantes, pero tiene el problema de que es más compleja de implementar en el contexto escolar y ello genera menores condiciones para una participación regular, y el desafío de manejar las situaciones de burlas y estigmatización a que pueden ser expuestas.

Esta investigación presenta diversas limitaciones. El tamaño de la muestra impidió controlar el efecto de pertenencia a distintas unidades educativas de las participantes. En particular, la modalidad universal presenta más acentuadas limitaciones metodológicas, por no estar aleatorizada la asignación al grupo control y experimental, y haber sido obtenido mayoritariamente al año siguiente. Pese a estas limitaciones, esta investigación constituye un útil 
antecedente para el desarrollo de iniciativas de prevención en adolescentes chilenas.

No es claro qué razones explican que las intervenciones, tanto indicadas como universales, en ocasiones muestren resultados positivos y en otros no (Spence \& Shortt, 2007). Esta falta de consistencia ha llevado al desarrollo de nuevas formas de intervención, más largas, intensivas y amplias. Sin embargo, sus resultados no han sido más positivos (Sawyer et al., 2011; Sheffield et al., 2006). Un desafío de la investigación es identificar qué factores serían más relevantes para el logro de mayor eficacia de las intervenciones.

\section{Referencias}

Beck, A. \& Steer, R. (1993). Beck anxiety inventory. San Antonio, TX: Psychological Corporation.

Beck, A., Steer, R. \& Brown, G. (1996). Beck depression inventory. San Antonio, TX: Psychological Corporation.

Bravo, M., Ribera, J., Rubio-Stipec, M., Canino, G., Shorout, P., Ramírez, R, Fábregas, L., Chávez, L., Alegría, M., Bauermeister, J.J. \& MartínezTaboas, A. (2001). Test-retest reliability of the spanish version of the diagnostic interview schedule for children (DISC-IV). Journal of Abnormal Child Psychology, 29, 433-444.

Calear, A. \& Christensen, H. (2010). Systematic review of school-based prevention and early intervention programs for depression. Journal of Adolescence 33, 429-438.

Clarke, G. \& Lewinsohn, P. (1995). Instructor's manual for the adolescent coping with stress course. Unpublished Manual. Oregon Health Sciences University. Portland: Oregon.

Collins, K. \& Dozois, D. (2008). What are the active ingredients in preventative interventions for depression? Clinical Psychology: Sciencie and Practice, 15, 313-330.

Cova, F. (2005). Una perspectiva evolutiva de las diferencias de género en los trastornos depresivos, Terapia Psicológica, 23, 49-58.

Cova, F., Aburto, B., Sepúlveda, M. J. \& Silva, M. (2006). Potencialidades y obstáculos de la prevención de la depresión en niños y adolescentes. Revista Psykhe, 15, 57-66.

Cova, F., Rincón, P. \& Melipillán, R. (2007b). Rumiación y presencia de sintomatología ansiosa y depresiva en adolescentes, Revista Mexicana de Psicología, 24, 175-183.

Chaplin, T., Gillham, J., Reivich, K., Elkon, A., Samuels B, Freres D., Winder B. \& Seligman M. (2006). Depression prevention for early adolescent girls. A pilot study of all girls versus co-ed groups. Journal of Early Adolescence 26, 110-126.

Garber, J. (2008). Prevention of depression: Are we there yet? Clinical Psychology-Science and Practice, 15, 336-341.

Gillham, J., Jaycox, L.J., Reivich, K.J., Hollon, S.D., Freeman, A., DeRubeis, R. J. \& Seligman, M. E. (1992). The apex project manual for group leaders. University of Pennsylvania: Philadelphia.

Gillham, J, Shatté, A. \& Freres, D. (2000). Preventing depression: A review of cognitive-behavioral and family intervention. Applied and Preventive Psychology, 9, 63-88.
Gladstone, T., Beardslee, W. \& O'Connor, E. (2011). The prevention of adolescent depression. Psychiatric Clinics of North America, 34, 35-52.

Hankin, B. \& Abramson, L. (2002). Measuring cognitive vulnerability to depression in adolescence: Reliability, validity, and gender differences. Journal of Clinical Child and Adolescent Psychology, 31, 491-504.

Horowitz, J. L. \& J. Garber (2006). The prevention of depressive symptoms in children and adolescents: A meta-analytic review. Journal of Consulting and Clinical Psychology 74, 401-415.

Le, H., Muñoz, R., Ippen, Ch. \& Stoddard, J. (2003). Treatment is not enough: We must prevent major depression in women. Prevention \& Treatment, 6, 1-6.

Melipillán, R., Cova, F., Rincón, P. \& Valdivia, M. (2008). Propiedades psicométricas del inventario de depresión de Beck-II en adolescentes chilenos. Terapia Psicológica, 26, 59-70.

Merry, S., Mc Dowell, H., Hetrick, S., Bir, J. \& Muller, N. (2003). Intervenciones psicológicas y educativas para la prevención de la depresión en niños y adolescentes. La Cochrane Library Plus en español. Oxford: Update Software.

Muñoz, R., Cuijpers, P., Smit, F., Barrera, A. \& Leykin, Y. (2010). Prevention on major depression. Annual Review of Clinical Psychology, 6, 181-212.

Rapee, R. M., Wignall, A., Sheffield, J., Kowalenko, N., Davis, A., McLoone, J. \& Spence, S. (2006). Adolescents' reactions to universal and indicated prevention programs for depression: Perceived stigma and consumer satisfaction. Prevention Science, 7, 167-177.

Rosenberg, A. (1965). Society and the adolescent self-image. New Jersey: Princeton.

Sanz J. \& Navarro, M.E. (2003). Propiedades psicométricas de una versión española del Inventario para la Ansiedad de Beck-II (BAI). Ansiedad y Estrés 9, 239-288.

Sawyer, M., Harchak, T., Spence, S., Bond, L., Graetz, B., Kay, D., Patton, G. \& Sheffield, J. (2010). School-based prevention of depression: A 2-year follow-up of a randomized controlled trial of the beyondblue schools research initiative. Journal of Adolescent Health 47, 297-304.

Sheffield, J. K., Spence, S. H., Rapee, R., Kowalenko, N., Wignall, A., Davis, A. \& McLoone, J.. (2006). Evaluation of universal, indicated, and combined cognitive-behavioral approaches to the prevention of depression among adolescents. Journal of Consulting and Clinical Psychology, 74, 66-79.

Shochet, I., Dadds, M., Holland, D., Whitefield, K., Harnett, P. \& Osgarby, S. (2001). Journal of Clinical Child Psychology, 30, 303-315

Spence, S. H. \& A. L. Shortt (2007). Research review: Can we justify the widespread dissemination of universal, school-based interventions for the prevention of depression among children and adolescents? Journal of Child Psychology and Psychiatry, 48, 526-542.

Treynor, W., Gonzalez, R. \& Nolen-Hoeksema (2003). Rumination reconsidered: A psychometric analysis. Cognitive Therapy and Research, 27, 247-259.

Zahn-Waxler, C., Crik, N., Shirtcliff, E. \& Woods, E. (2006). The origen and development of psychopathology in females and males. En D. Cichetti \& D. Cohen (Eds.). Developmental Psychopathology V. 1. Second Edition (76-138). New Jersey: Wiley and Sons. 